\title{
STARK EFFECT FOR DONORS IN DOUBLE QUANTUM WELLS
}

\section{S. ROLNIK AND J. ADAMOWSKI}

Faculty of Physics and Nuclear Techniques, Technical University of Mining and Metallurgy (AGH), Al. Mickiewicza 30, 30-059 Kraków, Poland

The problem of a hydrogenic donor in a semiconductor double quantum well under a homogeneous electric field is studied by variational means. The energy levels corresponding to the quantum states $1 s, 2 s, 2 p$ have been calculated with the help of the many-element variational basis consisting of the exponential functions. The influence of the electric field on the donor binding energy is determined for different impurity positions. The $1 s-2 p$ transition energies have been calculated for a possible comparison with experiment.

PACS numbers: $73.20 . \mathrm{Dx}, 73.20 . \mathrm{Hb}$

Among semiconductor heterostructures, the double quantum wells (DQW) have attracted much interest in the past few years, since they can serve as a starting point for studying the semiconductor superlattices. As it has been recently observed [1], an application of the electric field to the quantum well (QW) structures leads to the field-induced localization of electron states, which is manifested as the Stark ladder of electron energy levels. Doping such structures with impurities results in a considerable change in the electron charge distribution depending in which of the wells (with respect to the sign of the applied voltage) the attractive center is located. The influence of the electric field on the hydrogen-like donor quantum states for a single QW has been experimentally and theoretically studied by Yoo et al. [2] and Latge et al. [3]. The influence of the magnetic field on the donor states in the DQW structures has been investigated by Ranganathan et al. [4]. In the present paper, the properties of the donor states $(1 s, 2 s, 2 p)$ for the DQW under the static external electric field are determined by variational means. To the best of our knowledge, this effect has not been investigated by theoretical methods until now.

We consider a hydrogenic model of the donor impurity in the DQW. The system consists of the electron and the positive donor center located in one of the wells. The DQW semiconductor structure is described by the potential energy profile which consists of the two wells of the same depth and thickness (denoted by $V_{\mathrm{B}}$ and $L$, respectively) separated by the barrier of width $W$. We assume that the homogeneous electric field of the strength $F$ is applied to the DQW structure along the growth axis ( $z$ axis). The donor ionization by the field is neglected. Choosing 
the donor units, i.e., the donor rydberg $\left(R_{\mathrm{D}}\right)$ as a unit of energy and donor Bohr radius $\left(a_{\mathrm{D}}\right)$ as a unit of length, the Hamiltonian of our system in the effective mass approximation can be written as

$$
H=-\nabla^{2}-\frac{2}{r}+V(z)+F z
$$

where the electron-donor distance $r=\left[\rho^{2}+\left(z-z_{0}\right)^{2}\right]^{1 / 2}, \rho^{2}=x^{2}+y^{2}$, and $z_{0}$ is the donor center $z$-coordinate. We assume that $z_{0}$ is varied within the right well and the potential energy of the DQW is given by $V(z)=0$ for $W / 2<|z|<$ $L+W / 2$ and $V(z)=V_{\mathrm{B}}$ otherwise. The eigenvalue problem for Hamiltonian (1) has no analytical solutions, therefore, we apply the variational procedure in order to solve this problem. The trial wave function is proposed in the form of the linear combination

$$
\psi=\sum_{i} c_{i} \phi_{i}
$$

with the basis wave functions

$$
\phi_{i}=f(z) \mathrm{e}^{\mathrm{i} m \varphi} \rho^{|m|} \exp \left(-\alpha_{i} r\right) \exp \left(-\beta_{i} z\right),
$$

where $m$ is the magnetic quantum number, $f(z)$ is the exact solution of the Schrödinger equation for the double potential well, and $\alpha_{i}, \beta_{i}$, and $c_{i}$ are the variational parameters. The solution $f(z)$ can be found by the transfer matrix method [5] assuming that $f(z)$ and $\mathrm{d} f(z) / \mathrm{d} z$ are continuous across the interfaces. The energy eigenvalues $E_{n}$ of the donor states have been estimated by the minimalization of the expectation values of Hamiltonian (1) calculated with the use of trial wave function (2). The binding energy is defined with the respect to the ground state energy $E_{0}$ of the electron in the DQW structure without donor, i.e., $E_{\mathrm{B}}^{n}=E_{0}-E_{n}$, where the energy $E_{0}$ is calculated variationally assuming $\alpha_{i}=0$ in Eqs. (3).
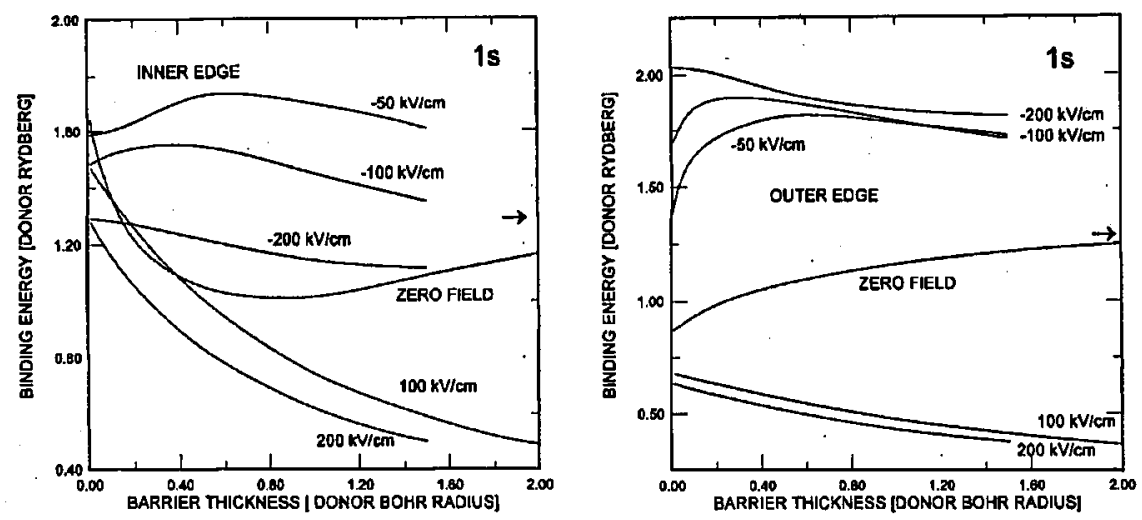

Fig. 1. Binding energy of the ground state for the donor at the inner-edge and outer-edge positions as a function of the barrier width for several values of the electric field. The arrows show the limit values for the single $\mathrm{QW}$. 

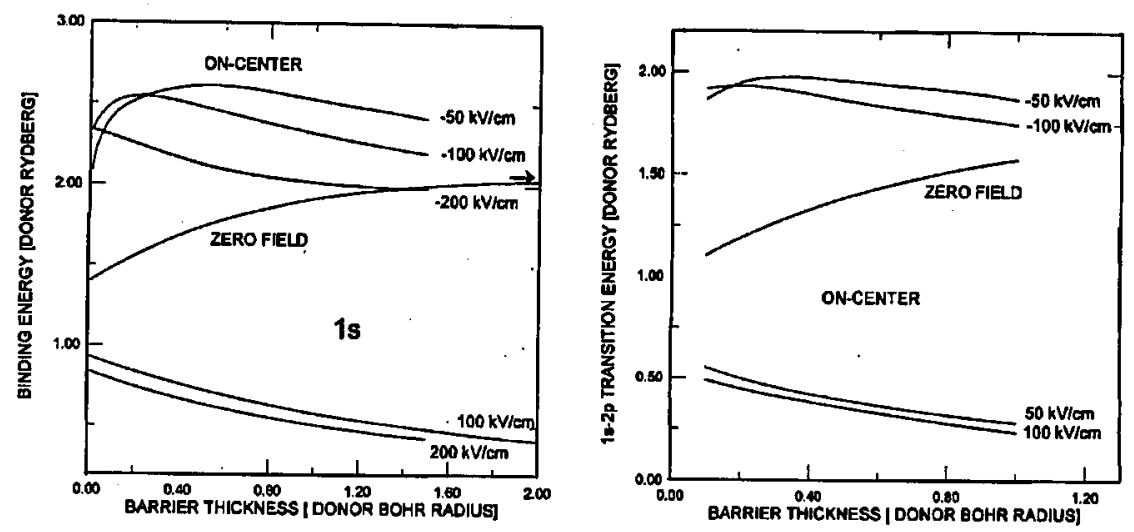

Fig. 2. Binding energy of the ground state for the on-center donor and $1 s-2 p$ transition energy as a function of the barrier width for several values of the electric field. The arrow shows the limit value for the single $\mathrm{QW}$.

We have performed the calculations for the three states of the lowest energy, which correspond to the $1 s, 2 s$, and $2 p$ states of the hydrogen-like donor. We used the following values of the parameters: $L=1 a_{\mathrm{B}} \cong 100 \AA, V_{\mathrm{B}}=50 R_{\mathrm{D}} \cong 300 \mathrm{meV}$, which are typical for the $\mathrm{Al}_{x} \mathrm{Ga}_{1-x} \mathrm{As} / \mathrm{GaAs}(x \cong 0.3)$ structure. Figures 1 and 2 show the results obtained for the ground state $(1 s)$ binding energy. For limiting case $(W \rightarrow 0)$ the present results agree with those [6] for the single QW.

In zero-field case the binding energy is the increasing function for the on-center and outer-edge positions (but takes on much larger values for the on-center position); however, for the inner-edge donor location, the binding energy has a minimum at the barrier thickness of about $1 a_{\mathrm{D}}$.

For the non-zero electric field, the essential difference can be observed in the dependence of the binding energy on the direction of the field. If the electric field is applied in the direction of the positive $z$ axis (the positive electric bias), the binding energy decreases as a function of electric field strength. This effect is due to the shifting of the electronic distribution by the electric field into the adjacent well with the lower potential energy of the electron in the electric field, but without the attractive donor center. For the negative electric bias, the binding energy is larger than for the zero-field case because the electron distribution is concentrated in the right well (with the attractive donor center). In this case, the influence of the applied electric field is different for the different donor position. For the inner-edge donor position, the binding energy is a decreasing function of the field for all barrier widths. However, for the outer-edge and on-center donor locations, the binding energy is a more complicated function of both the electric field and barrier width. For the $2 s$ state, we obtained the similar dependence as for $1 s$ but with the lower binding energy (about $0.2-0.3 R_{\mathrm{D}}$ ). The calculated $1 s-2 p$ transition energies shown in Fig. 2 can be helpful in a quantitive interpretation of the expected experimental results. 


\section{References}

[1] E.E. Mendez, F. Agullo-Rueda, J.M. Hong, Phys. Rev. Lett. 60, 2426 (1988).

[2] Byungsu Yoo, B.D. McCombe, W. Schaff, Phys. Rev. B 44, 13152 (1991).

[3] A. Latge, N. Porras-Montenegro, L.E. Oliveira, Phys. Rev. B 51, 2259 (1995).

[4] R. Ranganathan, B.D. McCombe, N. Nguyen, Y. Zhang, M.L. Rustgi, W.J. Schaff, Phys. Rev. B 44, 1423 (1991); ibid 44, 3344 (1991).

[5] N. Nguyen, J.X. Zang, R. Ranganathan, B.D. McCombe, M.L. Rustgi, Phys. Rev. $B$ 48, 14226 (1993).

[6] S. Rolnik, J. Adamowski, Ukr. J. Phys. 40, 680 (1995). 